\title{
AUTOIMMUNITY
}

\section{Glycoengineering has therapeutic potential}

Modulating antibody glycans in vivo is a potential new strategy for the treatment of autoimmune diseases, as revealed by new findings published in Cell. In the study, such an approach attenuated autoimmune disease in two mouse models of autoimmunity in both a prophylactic and a therapeutic fashion.

"Research over the past few years has provided strong evidence that immunoglobulin G ( $\operatorname{IgG}$ ) activity can be modulated by the presence or absence of certain sugar residues in the IgG Fc domain. In a variety of autoimmune and chronic inflammatory diseases the absence of sialic acid and galactose residues is associated with active disease and tissue inflammation," describes Falk Nimmerjahn, who was not involved in the study. "The anti-inflammatory activity of intravenous immunoglobulin (IVIG) therapy, which triggers resolution of inflammation, depends at least in part on sialylated IgG glycovariants," he continues.

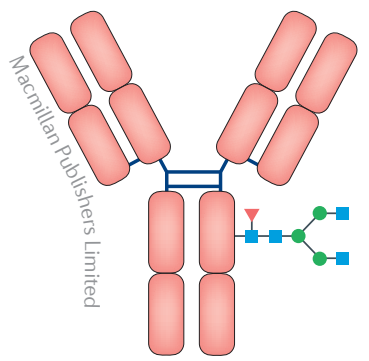

Pro-inflammatory antibody
4

Co-

administration

of soluble

sialic acid and

galactosyl

transferases

... attenuated

disease

activity in two

mouse models

of autoimmune

disease

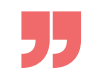

Engineered sialic acid and galactosyl transferase enzymes

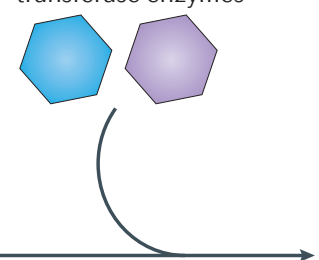

"Canonically, glycosylation is thought to occur exclusively in the endoplasmic reticulum and Golgi apparatus in the secretory pathway," explains Robert Anthony, corresponding author of the study. "Recent work has suggested that IgG sialylation can occur outside of the cell, by glycosylation enzymes released by hepatocytes. So, we set out to make hypersialylated IgG in vivo, by generating our own soluble glycosylation enzymes."

Co-administration of soluble sialic acid and galactosyl transferases fused to human IgG Fc domains, which attached sialic acid and galactose, respectively, to glycans, attenuated disease activity in two mouse models of autoimmune disease: arthritogenic sera-induced arthritis and a model of Goodpasture disease that results in nephrotoxic nephritis. The reduction in disease activity mirrored that observed with IVIG treatment and, when administered prophylactically, these enzymes were more effective than IVIG treatment at suppressing ongoing inflammation.

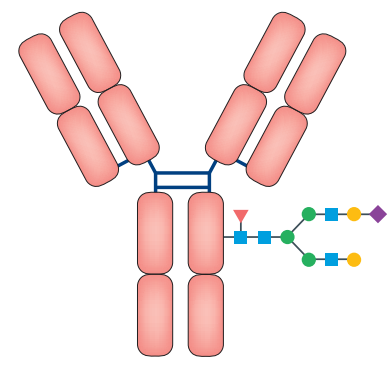

Anti-inflammatory antibody
The anti-inflammatory effects of these enzymes were dependent on CD209 antigen (also known as DC-SIGN), signal transducer and activator of transcription 6 (STAT6) signalling, and low-affinity immunoglobulin- $\gamma$ Fc region receptor IIb (Fc $\gamma$ RIIB), as shown with various knockout mouse strains. Removal of the Fc glycan from these engineered enzymes had no effect on their anti-inflammatory activity, indicating that the transferase activity and not the Fc glycans on the engineered enzymes was responsible for their anti-inflammatory effects.

"Intriguingly, sialylation was restricted to IgG at the site of inflammation, and did not occur systemically or off target," states Anthony. "This finding was rather serendipitous, but is attributable to two factors. Firstly, IgGs are unusual glycoproteins in that they predominantly lack sialic acid, and as a result can readily be sialylated (most glycoproteins are highly sialylated). Secondly, platelets recruited to the site of inflammation released sugar-nucleotide donors required for sialylation."

Anthony and colleagues found that treatment with clopidogrel, an inhibitor of platelet activation, prevented the anti-inflammatory effects of these enzymes in both mouse models. They also confirmed that in vitro human platelets released sialic acid and galactose nucleotide donors upon activation.

"It would be very interesting to know if administration of such enzymes would also be able to ameliorate other autoimmune diseases and most importantly if it also works on the background of a human immune system," says Nimmerjahn.

Jessica McHugh

ORIGINAL ARTICLE Pagan, J. D. et al.

Engineered sialylation of pathogenic antibodies in vivo attenuates autoimmune disease. Cell https://doi.org/10.1016/j.cell.2017.11.041 (2017) FURTHER READING Seeling, M. et al. Differential antibody glycosylation in autoimmunity: sweet biomarker or modulator of disease activity? Nat. Rev. Rheum. 13, 621-630 (2017) 